\title{
The degenerate distributive complex is degenerate
}

\author{
Józef H. Przytycki ${ }^{1,2}$ - Krzysztof K. Putyra ${ }^{3}$
}

Received: 15 September 2015 / Revised: 29 July 2016 / Accepted: 22 August 2016 /

Published online: 20 October 2016

(C) Springer International Publishing AG 2016

\begin{abstract}
We prove that the degenerate part of the distributive homology of a multispindle is determined by the normalized homology. In particular, when the multispindle is a quandle $Q$, the degenerate homology of $Q$ is completely determined by the quandle homology of $Q$. For this case (and generally for two-term homology of a spindle) we provide an explicit Künneth-type formula for the degenerate part. This solves the mystery in algebraic knot theory of the meaning of the degenerate quandle homology, brought over 15 years ago when the homology theories were defined, and the degenerate part was observed to be nontrivial.
\end{abstract}

Keywords Spindle · Quandle · Rack homology · Quandle homology · Degenerate homology

Mathematics Subject Classification 55N35 - 18G60

\footnotetext{
JHP was partially supported by the NSA-AMS 091111 Grant, the GWU-REF Grant, and the Simons Collaboration Grant 316446 . KKP was partially supported by the Columbia University Topology RTG Grant DMS-0739392 and by the NCCR SwissMAP.

$\bowtie \quad$ Krzysztof K. Putyra

krzysztof.putyra@eth-its.ethz.ch

Józef H. Przytycki

przytyck@gwu.edu

1 Department of Mathematics, The George Washington University, 801 22nd St NW, Washington, DC 20052, USA

2 Institute of Mathematics, University of Gdańsk, ul. Wita Stwosza 57, 80-308 Gdańsk, Poland

3 Institute for Theoretical Studies, ETHZ, Clausiusstrasse 47, 8092 Zürich, Switzerland
} 


\section{Introduction}

Quandle homology $[1,3,4,7]$ is built in analogy to group homology or Hochschild homology of associate structures. In the unital associative case we deal with simplicial sets (or modules) and it is a classical result that the degenerate part of a chain complex is acyclic, so that homology and normalized homology are isomorphic. It is not the case for distributive structures: we deal here only with a weak simplicial module [10] and the degenerate part can be not acyclic, as observed for quandles. It has been proven that the distributive homology of a quandle (called the rack homology) splits into degenerate and normalized parts [8] (the latter called the quandle homology), but no clear general connection between the two parts was observed.

Quandles are a special case of multispindles, sets with a number of self-distributive operations, for which analogous homology theory exists [10]. With a right definition of a module over a multispindle one can define homology with coefficients, generalizing twisted rack homology [2]. In this paper we construct a filtration on the degenerate chain complex with coefficients, obtaining a spectral sequence with a nice second page.

Theorem 5.4 Let a multispindle $X$ act on an $R$-module $M$. Then there is a spectral sequence $\left(E^{r}, \partial^{r}\right)$ converging to the degenerate multiterm homology $H^{\mathrm{D}}(M ; X)$ such that $E_{p q}^{2}=H_{p}^{\mathrm{N}}\left(\widehat{H}_{q-2}(M ; X) ; X\right)$.

Therefore, the degenerate homology in degree $q$ is controlled by homology in degree less than $q$. This motivates to look for some recursive formula computing degenerate homology from the normalized one, and as we shall see later such a formula exists at least for (one-term) homology of spindles and (two-term) homology of quandles.

In the first case we construct an explicit isomorphism between the degenerate chain complex and its associated graded complex with respect to the filtration mentioned above, resulting in an isomorphism

$$
H_{n}^{\mathrm{D}}\left(M ; X, \partial^{\star}\right) \cong \bigoplus_{p+q=n} \widehat{H}_{q-2}\left(M ; X, \partial^{\star}\right) \otimes C_{p}^{\mathrm{N}}(X)
$$

for any spindle $X$ (see Theorem 6.3). In particular, the spectral sequence mentioned above collapses at the first page. This leads to a recursive formula for degenerate homology, which can be easily solved. For instance, we obtain the following decomposition.

Corollary 6.4 If the spindle $(X, \star)$ is finite, then

$$
H_{n}^{\mathrm{D}}\left(X, \partial^{\star}\right) \cong \bigoplus_{p=1}^{n} \widetilde{H}_{n-p}^{\mathrm{N}}\left(X, \partial^{\star}\right)^{\oplus|X|\left(|X|^{p}-(-1)^{p}\right) /(1+|X|)} .
$$

In the case of rack homology the graded associated chain complex is not isomorphic to the degenerate complex - the first page on the spectral sequence has a nonvanishing 
differential. Instead, the degenerate chain complex $C^{\mathrm{D}}(M ; X)$ is an isomorphism to the tensor product $\widehat{C}(M ; X)[2] \otimes C^{\mathrm{N}}(X)$, leading to a Künneth-type formula, see Theorem 7.1 for the exact statement. The immediate corollary is that the normalized homology controls the degenerate part.

Corollary 7.2 Suppose a spindle homomorphism $\varphi: X \rightarrow X^{\prime}$ induces an isomorphism on the normalized rack homology $\varphi_{*}: H^{\mathrm{N}}\left(X, \partial^{\mathrm{R}}\right) \rightarrow H^{\mathrm{N}}\left(X^{\prime}, \partial^{\mathrm{R}}\right)$. If $M$ is an $X^{\prime}-$ module such that the induced map $\varphi_{*}: H^{\mathrm{N}}\left(M^{\varphi} ; X, \partial^{\mathrm{R}}\right) \rightarrow H^{\mathrm{N}}\left(M ; X^{\prime}, \partial^{\mathrm{R}}\right)$ is also an isomorphism, so is $\varphi_{*}: H^{\mathrm{D}}\left(M^{\varphi} ; X, \partial^{\mathrm{R}}\right) \rightarrow H^{\mathrm{D}}\left(M ; X^{\prime}, \partial^{\mathrm{R}}\right)$.

It is not immediately clear that the same should hold for homology of all multispindles, even for those with two nontrivial operations. The best we could achieve is that if a homomorphism of multispindles induces an isomorphism on normalized homology with coefficients in any module from a certain class, then it induces an isomorphism on degenerate homology.

Theorem 5.8 Choose multispindles $X, X^{\prime}$, an $X^{\prime}$-module $M$, and a multispindle homomorphism $\varphi: X \rightarrow X^{\prime}$ inducing an isomorphism $\varphi_{*}: H^{\mathrm{N}}\left(M^{\varphi} ; X\right) \rightarrow H^{\mathrm{N}}\left(M ; X^{\prime}\right)$. If it also induces an isomorphism $\varphi_{*}: H^{\mathrm{N}}\left(N^{\varphi} ; X\right) \rightarrow H^{\mathrm{N}}\left(N ; X^{\prime}\right)$ for any $X^{\prime}$-module $N$ with a vanishing compound action, then $\varphi_{*}: H^{\mathrm{D}}\left(M^{\varphi} ; X\right) \rightarrow H^{\mathrm{D}}\left(M ; X^{\prime}\right)$ is an isomorphism.

We prove it by showing that such a homomorphism induces an isomorphism on the second page of the spectral sequence. The inductive argument is based on the following result on spectral sequences, which we have not encountered before.

Lemma 8.8 Assume there is a homomorphism of spectral sequences $f_{p q}^{r}: E_{p q}^{r} \rightarrow \bar{E}_{p q}^{r}$ such that $f_{p q}^{2}$ is an isomorphism for $q \leqslant N$. Then $f_{p q}^{\infty}: E_{p q}^{\infty} \rightarrow \bar{E}_{p q}^{\infty}$ is an isomorphism for $p+q=N$.

As the proof is very technical and not related directly to distributive homology, we decided to move it to the end of the paper.

Outline. The paper is organized as follows. We provide basic definitions and results on distributive and rack homology in Sect. 2, and homology with coefficients in modules over mutlispindles is introduced in Sect. 3. Most of the computation is done using a graphical calculus explained briefly in Sect. 4 . The next three sections contain the main results of the paper: construction of the spectral sequence for degenerate homology in Sect. 5, and the analysis of the special cases of one-term (Sect. 6) and rack homology (Sect. 7). We provide a very brief discussion on spectral sequences in Appendix, including the proof of the technical Lemma 8.8.

\section{Distributive and rack homology}

Definition 2.1 A spindle is a set $X$ with a binary operation $\star: X \times X \rightarrow X$ that is idempotent and self-distributive from the right side, i.e. $x \star x=x$ and $(x \star y) \star z=$ 
$(x \star z) \star(y \star z)$ for any $x, y, z \in X$. If the function $x \mapsto x \star y$ is invertible for any $y \in X$ we say that $(X, \star)$ is a quandle. By dropping the idempotent condition, we obtain respectively a shelf and a rack.

The names shelf and spindle were coined by Crans in her $\mathrm{PhD}$ thesis [5] and are broadly used by knot theorists. The older names, used outside knot theory, are respectively a right distributive system and a right distributive idempotent system, see [6].

Definition 2.2 Choose a set $X$ with a number of spindle operations $\star_{1}, \ldots, \star_{r}$. We say that $\left(X ; \star_{1}, \ldots, \star_{r}\right)$ is a multispindle if the operations are mutually distributive, i.e. $\left(x \star_{i} y\right) \star_{j} z=\left(x \star_{j} z\right) \star_{i}\left(y \star_{j} z\right)$ for any $x, y, z \in X$. We define multishelves likewise.

Remark 2.3 As the trivial operation $x \vdash y=x$ is distributive with respect to any shelf operation $[10,11]$, one can extend any shelf $(X, \star)$ to a mutlishelf $(X ; \star, \vdash)$.

Given a spindle $(X, \star)$ and a ring $R$, we define $C_{n}(X)=R\left\langle X^{n+1}\right\rangle$ to be the $R$ module generated freely by all sequences $\left(x_{n}, \ldots, x_{0}\right) \in X^{n+1}$. We shall write $\underline{x}$ for such a sequence and we define $|\underline{x}|=n$. The (one-term) distributive differential $\partial^{\star}: C_{n}(X) \rightarrow C_{n-1}(X)$ is given as the alternating sum of face maps $d_{i}^{\star}: C_{n}(X) \rightarrow$ $C_{n-1}(X)$ :

$$
\begin{aligned}
& \partial^{\star}=\sum_{i=0}^{n}(-1)^{n-i} d_{i}^{\star}, \\
& d_{i}^{\star}\left(x_{n}, \ldots, x_{0}\right)=\left(x_{n} \star x_{i}, \ldots, x_{i+1} \star x_{i}, x_{i-1}, \ldots, x_{0}\right) .
\end{aligned}
$$

The unusual sign convention for $\partial^{\star}$ is the result of enumerating elements in a sequence $\underline{x}$ from right to left, contrary to the standard practice $[3,4,10,12]$. We check the presimplicial relation $d_{i}^{\star} d_{j}^{\star}=d_{j-1}^{\star} d_{i}^{\star}$ for $i>j$ (see also Example 4.1), from which it follows $\left(\partial^{\star}\right)^{2}=0$. We call the homology of this chain complex the (one-term) distributive homology of $X$.

Given a multishelf $\left(X ; \star_{1}, \ldots, \star_{r}\right)$, one can check that $\partial^{\star_{i}} \partial^{\star_{j}}+\partial^{\star_{j}} \partial^{\star_{i}}=0$. This guarantees that any linear combination $\partial=\sum_{k=1}^{r} a_{k} \partial^{\star_{k}}$ is a differential on $C(X)$, which we call the multiterm distributive differential with weights $\left(a_{1}, \ldots, a_{r}\right)$. Of particular interest is the case of the rack differential $\partial^{\mathrm{R}}=\partial^{\vdash}-\partial^{\star}$, where $x \vdash y=x$ $[4,7]$. Notice that our rack homology is shifted by one comparing to the definition due to Fenn, Rourke, and Sanderson. The main reason is that we wanted to deal with a pre-simplicial category while they chose the convention of a pre-cubic category.

Remark 2.4 We shall write $C(X)$ for the chain complex and $H(X)$ for its homology if we do not want to specialize the differential. Otherwise, the notation $C\left(X, \partial^{\star}\right)$, $H\left(X, \partial^{\mathrm{R}}\right)$, etc. will be used.

Definition 2.5 Let $X$ be a multispindle. The degenerate chain complex $C^{\mathrm{D}}(X)$ is the subcomplex of $C(X)$ generated by sequences $\underline{x}$ with repetitions, i.e. $x_{i}=x_{i+1}$ for some $i$. The quotient $C^{\mathrm{N}}(X)=C(X) / C^{\mathrm{D}}(X)$ is called the normalized complex of $X$. Homologies of the complexes are called respectively degenerate and normalized, and they are denoted by $H^{\mathrm{D}}(X)$ and $H^{\mathrm{N}}(X)$. 
Remark 2.6 In case of quandles, the normalized homology is usually referred to as the quandle homology and is written as $H^{\mathrm{Q}}(X)$, see [4].

The following theorem was first proven for the rack homology of quandles [8], and then extended to the one-term and multiterm cases [10].

Theorem 2.7 ([8,10]) Given a multispindle X, the following short exact sequence:

$$
0 \longrightarrow C^{\mathrm{D}}(X) \longrightarrow C(X) \longrightarrow C^{\mathrm{N}}(X) \longrightarrow 0
$$

splits in a canonical way. In particular, $H(X) \cong H^{\mathrm{N}}(X) \oplus H^{\mathrm{D}}(X)$.

In the case of rack homology, sequences $\underline{x}$ with late repetitions (i.e. $x_{i}=x_{i-1}$ for some $i<n$ in $\left.\left(x_{n}, \ldots, x_{0}\right)\right)$ form a chain subcomplex $C^{\mathrm{L}}\left(X, \partial^{\mathrm{R}}\right) \subset C^{\mathrm{D}}\left(X, \partial^{\mathrm{R}}\right)$, which is a direct summand. It is called the late degenerate complex in [8].

Theorem 2.8 ([8]) There is a short exact sequence that splits canonically

$$
0 \longrightarrow C^{\mathrm{L}}\left(X, \partial^{\mathrm{R}}\right) \longrightarrow C^{\mathrm{D}}\left(X, \partial^{\mathrm{R}}\right) \longrightarrow C^{\mathrm{N}}\left(X, \partial^{\mathrm{R}}\right)[1] \longrightarrow 0 .
$$

In particular, $H_{n}^{\mathrm{N}}\left(X, \partial^{\mathrm{R}}\right)$ is a direct summand of $H_{n+1}^{\mathrm{D}}\left(X, \partial^{\mathrm{R}}\right)$.

The number in brackets indicates a homological shift: $C[k]_{n}=C_{n-k}$.

In the general case of multiterm homology, $C^{\mathrm{L}}(X)$ is a subcomplex of $C^{\mathrm{D}}(X)$ if and only if the sum of all weights is zero, in which case the proof from [8] of the theorem above holds. We shall generalize it to homology with coefficients in the next section.

\section{Homology with coefficients}

The following definition is motivated by the notion of an $X$-set, introduced by Kamada.

Definition 3.1 An action of a spindle $(X, \star)$ on an $R$-module $M$ is a function $\star: M \times X \rightarrow M$ that is linear in the first variable and

$$
(m \star x) \star y=(m \star y) \star(x \star y)
$$

for any $m \in M$ and $x, y \in X$. If $(X, \star)$ is a quandle we require also that it acts by automorphisms of $M$, i.e. the function $m \mapsto m \star x$ is invertible for every $x \in X$.

An $R$-module $M$ carrying an action of a spindle $X$ will be often called an $X$-module.

Example 3.2 Consider a spindle $(X, \vdash)$ with the trivial operation $x \vdash y=x$. Then the actions on $M$ of all elements of $X$ commute:

$$
(m \vdash x) \vdash y=(m \vdash y) \vdash(x \vdash y)=(m \vdash y) \vdash x .
$$

Hence, $X$-modules are precisely modules over the polynomial algebra $\mathbb{Z}[X]$ with as many variables as there are elements in $X$. 
We generalize Definition 3.1 to multispindles in a natural way.

Definition 3.3 An action of a multispindle $\left(X ; \star_{1}, \ldots, \star_{r}\right)$ on an $R$-module $M$ consists of functions $\star_{i}: M \times X \rightarrow M$ linear in the first variable, such that

$$
\left(m \star_{i} x\right) \star_{j} y=\left(m \star_{j} y\right) \star_{i}\left(x \star_{j} y\right)
$$

for any $m \in M, x, y \in X$ and $i, j=1, \ldots, r$.

Given a multispindle $\left(X ; \star_{1}, \ldots, \star_{r}\right)$, which acts on $M$, choose $a_{1}, \ldots, a_{r} \in R$. We define the compound action of $X$ on $M$ with weights $\left(a_{1}, \ldots, a_{r}\right)$ as the linear combination

$$
(m, x) \mapsto m \cdot x=\sum_{i=1}^{r} a_{i}\left(m \star_{i} x\right)
$$

A direct computation shows that the compound action is distributive with respect to the action of $X$ on $M$, i.e. $(m \cdot x) \star_{i} y=\left(m \star_{i} y\right) \cdot\left(x \star_{i} y\right)$ for $i=1, \ldots, r$.

Example 3.4 Every multispindle $X$ acts on any module $M$ trivially, $m \star_{i} x=m$, in which case the compound action is the multiplication by the sum of weights.

Example 3.5 A multispindle $X$ acts on the distributive chain complex $C(X)$ by acting on each element of a sequence: $\left(x_{n}, \ldots, x_{0}\right) \star_{i} y=\left(x_{n} \star_{i} y, \ldots, x_{0} \star_{i} y\right)$. This action descends to homology and it was observed in [11] that $\alpha \cdot w=0$ for any homology class $\alpha \in H(X)$ and $w \in X$. Indeed, $\alpha \cdot w=\partial h^{w}+h^{w} \partial$ for $h^{w}(\underline{x})=(-1)^{|\underline{x}|}(\underline{x}, w)$.

Let a multispindle $X$ act on an $R$-module $M$. We define the homology with coefficients in $M$ by setting $C_{n}(M ; X)=M \otimes C_{n}(X)$ and $d_{i}^{\star_{k}}(m \otimes \underline{x})=\left(m \star_{k} x_{i}\right) \otimes d_{i}^{\star_{k}} \underline{x}$. Clearly, $C(X)=C(R ; X)$ with the trivial action of $X$ on $R$. As before, we have the degenerate subcomplex with $C_{n}^{\mathrm{D}}(M ; X)=M \otimes C_{n}^{\mathrm{D}}(X)$ and the normalized one $C^{\mathrm{N}}(M ; X)=$ $C(M ; X) / C^{\mathrm{D}}(M ; X)$.

Proposition 3.6 The short exact sequence splits canonically

$$
0 \longrightarrow C^{\mathrm{D}}(M ; X) \longrightarrow C(M ; X) \longrightarrow C^{\mathrm{N}}(M ; X) \longrightarrow 0 .
$$

In particular, $H(M ; X) \cong H^{\mathrm{N}}(M ; X) \oplus H^{\mathrm{D}}(M ; X)$.

Proof Let $\alpha: C^{\mathrm{N}}(X) \rightarrow C(X)$ be the splitting map from Theorem 2.7. As each $C_{n}(X)$ is a free $R$-module, $C_{n}^{\mathrm{N}}(M ; X)=M \otimes C_{n}^{\mathrm{N}}(X)$ and an easy computation shows the map $\alpha^{M}=\mathrm{id} \otimes \alpha$ splits sequence (1).

The case of modules $M$ with a vanishing compound action is very special. As before let $C_{n}^{\mathrm{L}}(M ; X)=M \otimes C_{n}^{\mathrm{L}}(X)$ be spanned by late degenerate sequences. 
Proposition 3.7 If the compound action of $X$ annihilates $M$, then $C^{\mathrm{L}}(M ; X)$ is a subcomplex of $C^{\mathrm{D}}(M ; X)$ and there is an isomorphism $s: C^{\mathrm{N}}(M ; X)[1] \rightarrow$ $C^{\mathrm{D}}(M ; X) / C^{\mathrm{L}}(M ; X)$ given by the formula $s\left(m \otimes\left(x_{n}, \ldots x_{0}\right)\right)=(-1)^{n} m \otimes$ $\left(x_{n}, x_{n}, \ldots, x_{0}\right)$.

Proof Vanishing of the compound action implies vanishing of the compound face $\operatorname{map} d_{n}: C_{n}(M ; X) \rightarrow C_{n-1}(M ; X), d_{n}=\sum_{k} a_{k} d_{n}^{\star_{k}}$. Hence, $C^{\mathrm{L}}(M ; X)$ is a subcomplex of $C^{\mathrm{D}}(M ; X)$. For $s$ to be an isomorphism we have to check it is a chain map, which follows from the equality $d_{n}^{\star_{k}}=d_{n-1}^{\star_{k}}$ on $C_{n}^{\mathrm{D}}(M ; X) / C^{\mathrm{L}}(M ; X)$. Indeed, every sequence in the quotient $C^{\mathrm{D}}(M ; X) / C^{\mathrm{L}}(M ; X)$ begins with a repetition.

Remark 3.8 One can follow the proof of Theorem 2.8 from [8] to see that $C^{\mathrm{L}}(M ; X)$ is a direct summand of $C^{\mathrm{D}}(M ; X)$.

Remark 3.9 A multispindle $X$ acts naturally on its homology with coefficients. The compound action vanishes, which is proven using the homotopy $h^{w}(m \otimes \underline{x})=$ $(-1)^{|\underline{x}|} m \otimes(\underline{x}, w)$.

The one-term and multiterm chain complexes can be augmented with $C_{0}(X) \stackrel{\epsilon}{\rightarrow} R$ sending each generator to $\epsilon(x)=1$. This new chain complex and its homology are written as $\widetilde{C}(X)$ and $\widetilde{H}(X)$ respectively. We redefine it for complexes with coefficients in an $X$-module by appending to the chain complex $C(M ; X)$ the linearized compound action $C_{0}(M ; X)=M \otimes R\langle X\rangle \rightarrow M$. We write $\widehat{C}(M ; X)$ and $\widehat{H}(M ; X)$ for this chain complex and its homology respectively.

Remark $3.10 \widehat{H}_{n}(M ; X)=H_{n}(M ; X)$ for $n \geqslant 0$ and $\widehat{H}_{-1}(M ; X)=M$ when the compound action vanishes. In particular, $\widehat{H}(R ; X)$ is different from $\widetilde{H}(R ; X)$ in case of rack homology.

We construct the augmented normalized complex $\widehat{C}^{\mathrm{N}}(M ; X)$ with normalized homology $\widehat{H}^{\mathrm{N}}(M ; X)$ likewise. It is worth to notice that Proposition 3.6 still holds for augmented complexes.

\section{Graphical calculus}

Consider a picture in a plane consisting of a number of strands originating on a horizontal line and going upwards to another horizontal line. The strands can cross with each other ${ }^{1}$ and a single strand can terminate in a dot but there are no turnbacks. Given a spindle $(X, \star)$, we can interpret such a picture as a map $X^{n} \rightarrow X^{m}$, where $n$ and $m$ are numbers of endpoints of strands at the lower and upper horizontal lines respectively. Namely, decorate the $n$ endpoints on the lower line with elements of $X$ and propagate the labels upwards along the strands. A label is forgotten at a terminal dot, and each time we encounter a crossing the right label propagates with no change but the left one is replaced by the product of both, see Fig. 1 .

\footnotetext{
1 These are real crossings on a plane but we can interpret them with no harm as negative crossings.
} 

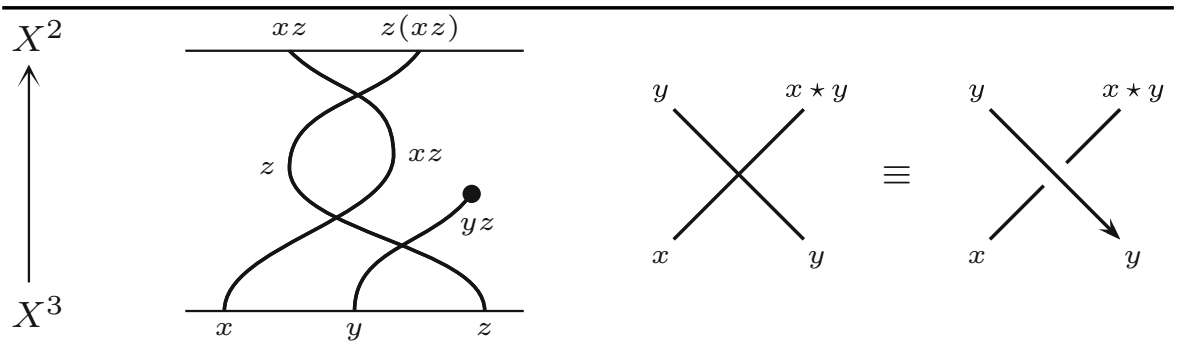

Fig. 1 A string diagram seen as a function $X^{3} \rightarrow X^{2}$. The pictures to the right explain how labels propagate through a crossing and where the motivation comes from

Different pictures can encode the same map. For instance, far away crossings and terminal dots commute:

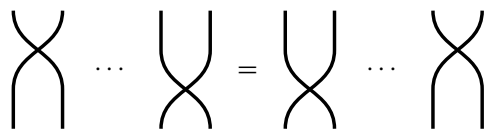

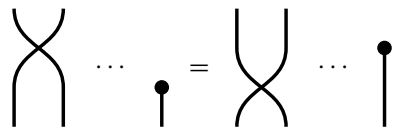

etc.

and a terminal dot can be pulled through a crossing from the left hand side but not from the right one:

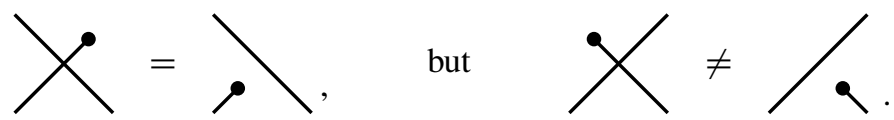

The most interesting are the following two relations:

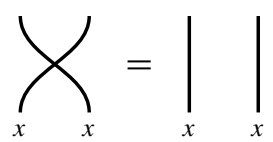

and

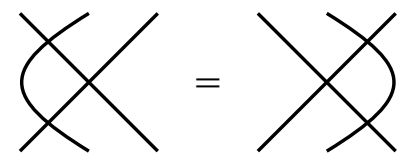

which are equivalent to the axioms of a spindle. The first one holds only when both inputs are labeled with the same element, and it visualizes the idempotency axiom. The right picture is the famous Reidemeister III move, also called the braid or the YangBaxter relation. It holds for any input and is equivalent to the self-distributivity of $\star: X \times X \rightarrow X$.

If $X$ acts on a module $M$, add a wall to the left of the picture-it can be labeled with elements $m \in M$. A strand can terminate on the wall, which corresponds to the action map $M \times X \rightarrow M$. The condition for an action of $X$ translates as absorbing a crossing by the wall:

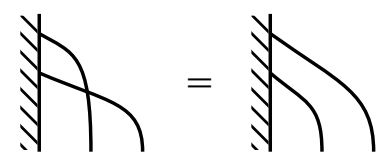

Example 4.1 The $i$-th face map $d_{i}^{\star}: C_{n}(M ; X) \rightarrow C_{n-1}(M ; X)$ can be visualized graphically by pulling the $i$-th strand all the way to the left, and terminating it at the wall: 


$$
d_{i}^{\star}=\left.\overbrace{n}\right|_{i}
$$

The presimplicial relation is shown by deforming pictures using relations (2), (4), and (5). For instance,

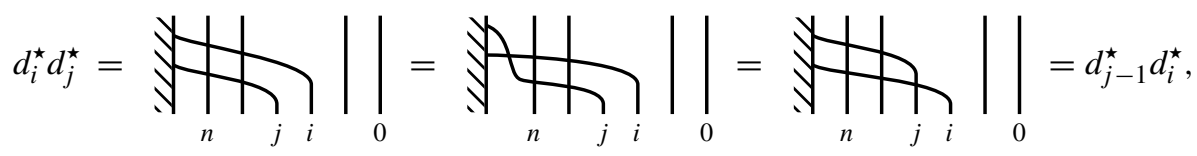

when $j>i$.

Example 4.2 In the case of the trivial operation $x \vdash y=x$, we can use a simpler picture for the face map $d_{i}^{\vdash}$ : the $i$-th strand is immediately terminated with a dot:

$$
d_{i}^{\vdash}=\left.y_{n}||_{i}\right|_{0}
$$

The presimplicial relation follows trivially, and in the mixed case we use (3). For instance, when $j>i$, one computes

$$
d_{i}^{\star} d_{j}^{\vdash}=\left.\left.\sqrt[y]{ }\right|_{n}\right|_{j}|=\sqrt{ }|_{0}=\left.\left.\right|_{n}\right|_{i}=d_{j-1}^{\vdash} d_{i}^{\star} .
$$

We shall often restrict to sequences with a repetition at a certain place, which will be visualized by a band joining two strands: it forces its two edges to carry the same label. For instance, we can visualize generators of $C^{\mathrm{D}}(M ; X)$ by vertical lines with a band at some position:

$$
C_{n}^{\mathrm{D}}(M ; X)=\left\langle\left.\Downarrow\right|_{n}||_{i}||_{0}: 0 \leqslant i<n\right\rangle,
$$

and the idempotency axiom can be rewritten without specifying labels at the input as

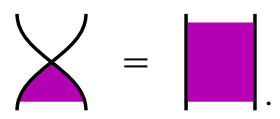

Strands that are not joined by a band can still carry the same label, however. Finally, the map $s_{p}: C_{p}(M ; X) \rightarrow C_{p+1}(M ; X)$ can be visualized by thickening the left most strand into a band

$$
s_{p}=(-1)^{p} \Downarrow \bigcup_{p}||_{i}||_{0}
$$


Remark 4.3 We shall use the same graphical calculus for multispindles without mixing different operations, i.e. we shall always use one operation to interpret a diagram.

\section{A filtration of the degenerate complex}

Let $\mathcal{F}_{n}^{p} \subset C_{n}^{\mathrm{D}}(M ; X)$ be the submodule generated by elements $m \otimes \underline{x}$, where the sequence $\underline{x}$ has a repetition at position $p$ or closer to the right, i.e. $x_{i}=x_{i+1}$ for some $i \leqslant p$. Diagrammatically,

$$
\mathcal{F}_{n}^{p}=\left\langle\left.\Downarrow\right|_{n}||_{i}||_{0}: 0 \leqslant i \leqslant p\right\rangle .
$$

Proposition 5.1 Each $\mathcal{F}^{p}$ is a chain subcomplex of $C^{\mathrm{D}}(M ; X)$, and all together they form a filtration of $C^{\mathrm{D}}(M ; X)$, i.e. $\mathcal{F}^{p} \subset \mathcal{F}^{p+1}$ for every $p$, and $\bigcup_{p \geqslant 0} \mathcal{F}^{p}=$ $C^{\mathrm{D}}(M ; X)$.

Proof The two conditions for a filtration follow directly from the definition of $\mathcal{F}_{n}^{p}$. To show that $\mathcal{F}^{p}$ is closed under the differential, choose a sequence $\underline{x}$ with a repetition at position $i$. Then for any operation $\star_{k}$ on $X$ the faces $d_{i}^{\star_{k}}(m \otimes \underline{x})=d_{i+1}^{\star_{k}}(m \otimes \underline{x})$ cancel each other, whereas the other faces have repetitions at positions $i$ or $i-1$ :
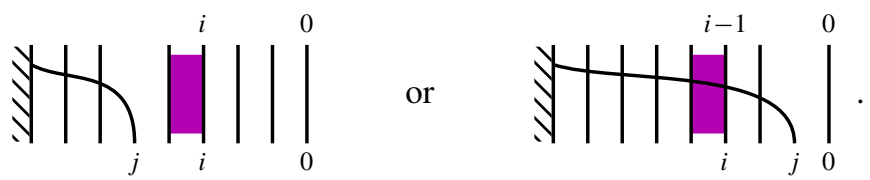

As the filtration is given on generators, the quotients $\mathcal{F}^{p} / \mathcal{F}^{p-1}$ are easy to understand: each is generated by elements $m \otimes \underline{x}$, where the sequence $\underline{x}$ has its first repetition occurring at the position $p$, when looking from the right hand side. As $d_{i}^{\star k}(m \otimes \underline{x}) \in$ $\mathcal{F}^{p-1}$ if $i<p$, only higher faces survive.

Corollary 5.2 There is an isomorphism of chain complexes

$$
\mathcal{F}^{p} / \mathcal{F}^{p-1} \cong \widehat{C}(M ; X)[p+2] \otimes C_{p}^{\mathrm{N}}(X),
$$

where on the right hand side the differential acts only on the first factor.

Proof The face maps $d_{i}^{\star_{k}}$ vanish on the quotient for $i<p$, and since $d_{p}^{\star_{k}}$ cancels $d_{p+1}^{\star k}$,

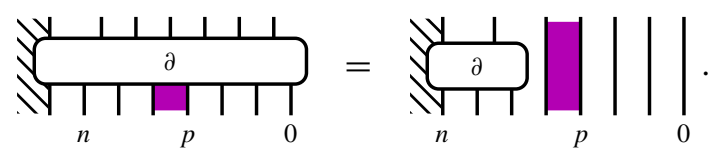

Hence, the repetition splits $\underline{x}$ into two parts: the left one, which can consist only of the wall (thence the augmented complex in (6)), and the right one (including the $p$-th strand), which has no repetition. The latter is preserved by the differential. 
Recall that the graded associated chain complex $\operatorname{gr} C^{\mathrm{D}}(M ; X)$ with respect to the filtration $\mathcal{F}^{p}$ is the direct sum of quotients $\mathcal{F}^{p} / \mathcal{F}^{p-1}$.

Corollary 5.3 Let $\left(X ; \star_{1}, \ldots, \star_{r}\right)$ be a multispindle acting on a module $M$. Then

$$
H_{n}\left(\operatorname{gr} C^{\mathrm{D}}(M ; X)\right) \cong \bigoplus_{p+q=n} \widehat{H}_{q-2}(M ; X) \otimes C_{p}^{\mathrm{N}}(X) .
$$

The filtration $\mathcal{F}^{p}$ leads to a spectral sequence (see Appendix) computing the degenerate homology of $X$, and the corollary above shows its first page. Its second page is the normalized homology of $X$ with coefficients in the augmented homology.

Theorem 5.4 Let a multispindle $X$ act on an $R$-module $M$. Then there is a spectral sequence $\left(E^{r}, \partial^{r}\right)$ converging to the degenerate multiterm homology $H^{\mathrm{D}}(M ; X)$ such that $E_{p q}^{2}=H_{p}^{\mathrm{N}}\left(\widehat{H}_{q-2}(M ; X) ; X\right)$.

Proof Due to Corollary 5.2, there is an isomorphism

$$
\operatorname{id} \otimes s_{p}: \widehat{H}_{q-2}(M ; X) \otimes C_{p}^{\mathrm{N}}(X) \stackrel{\cong}{\longrightarrow} E_{p q}^{1},
$$

so that the differential $\partial_{p q}^{1}: E_{p q}^{1} \rightarrow E_{p-1, q}^{1}$ computes homology of $s C^{\mathrm{N}}(X) \cong$ $C^{\mathrm{D}}(X) / C^{\mathrm{L}}(X)$ with coefficients in $\widehat{H}_{q-2}(M ; X)$. Since the compound action of $X$ on $\widehat{H}_{q-2}(M ; X)$ vanishes,

$$
s: C^{\mathrm{N}}\left(\widehat{H}_{q-2}(M ; X) ; X\right) \rightarrow s C^{\mathrm{N}}\left(\widehat{H}_{q-2}(M ; X) ; X\right)
$$

is actually a chain map, thence an isomorphism.

Corollary 5.5 Assume $\widehat{H}^{\mathrm{N}}(M ; X)=0$. Then degenerate homology vanishes. In particular, if the augmented homology of a spindle is trivial, so is its degenerate homology.

Proof Suppose $H_{i}^{\mathrm{D}}(M ; X)=0$ for $i<N$. Then $E_{p q}^{r}=H_{p}^{\mathrm{N}}\left(\widehat{H}_{q-2}(M ; X) ; X\right)=0$ for $q<N+2$. In particular, we have $E_{p q}^{\infty}=0$ when $p+q=N$, which implies $H_{N}^{\mathrm{D}}(M ; X)=0$.

Corollary 5.6 Assume the augmented quandle homology (i.e. $\partial=\partial^{-1}-\partial^{\star}$ ) of a spindle $X$ is trivial. Then $H_{n}^{\mathrm{D}}(R ; X)=R$ for $n>0$.

Proof The compound action on $R$ vanishes, so that $\widehat{H}_{0}^{\mathrm{N}}(R ; X)=\widehat{H}_{-1}^{\mathrm{N}}(R ; X)=R$ and $\widehat{H}_{p}^{\mathrm{N}}(R ; X)=H_{p}^{\mathrm{N}}(R ; X)=0$ for $p>0$. Hence, $E_{1,0}^{2}=0$ and $E_{0,1}^{2}=R$, which implies $H_{1}^{\mathrm{D}}(R ; X)=E_{0,1}^{2}=R$. Suppose by induction that $H_{i}^{\mathrm{D}}(R ; X)=R$ for $i=1, \ldots, N-1$. Then $E_{p q}^{2}=H_{p}^{\mathrm{N}}(R ; X)$ for $q<N+2$, which is zero except $E_{0, q}^{2}=H_{0}(R ; X)=R$. Again, it must be $H_{N}^{\mathrm{D}}(R ; X)=E_{0, N}^{2}=R$.

Choose a homomorphism of spindles $\varphi: X \rightarrow X^{\prime}$ and an $X^{\prime}$-module $M$. Then $\varphi$ induces an action of $X$ on $M, m \star_{i} x=m \star_{i} \varphi(x)$, and we shall write $M^{\varphi}$ for this $X$-module. 
Corollary 5.7 Suppose a homomorphism of multispindles $\varphi: X \rightarrow X^{\prime}$ induces isomorphisms on homology groups $\varphi_{*}: H^{\mathrm{N}}\left(\widehat{H}_{q}\left(M^{\varphi} ; X\right) ; X\right) \rightarrow H^{\mathrm{N}}\left(\widehat{H}_{q}\left(M ; X^{\prime}\right) ; X^{\prime}\right)$ for any $q$. Then $\varphi_{*}: H^{\mathrm{D}}\left(M^{\varphi} ; X\right) \rightarrow H^{\mathrm{D}}\left(M ; X^{\prime}\right)$ is an isomorphism.

Assume that $\varphi: X \rightarrow X^{\prime}$ given as above induces also an isomorphism on the normalized homology $\varphi_{*}: H^{\mathrm{N}}\left(M^{\varphi} ; X\right) \rightarrow H^{\mathrm{N}}\left(M ; X^{\prime}\right)$. Then $H\left(M^{\varphi} ; X\right) \cong H\left(M ; X^{\prime}\right)^{\varphi}$ as $X$-modules and we can restate the assumptions on $\varphi$ requiring that it induces an isomorphism on the normalized homology with coefficients in any module with a vanishing compound action. Since only $\widehat{H}_{k}(M ; X)$ with $k \leqslant n-2$ appear in $E_{p q}^{2}$ with $p+q=n$, one should be able to recover the original assumption of Corollary 5.7 by an induction argument. This is the idea underlying the following theorem.

Theorem 5.8 Choose multispindles $X, X^{\prime}$, an $X^{\prime}$-module $M$, and a multispindle homomorphism $\varphi: X \rightarrow X^{\prime}$ inducing an isomorphism $\varphi_{*}: H^{\mathrm{N}}\left(M^{\varphi} ; X\right) \rightarrow H^{\mathrm{N}}\left(M ; X^{\prime}\right)$. If it also induces an isomorphism $\varphi_{*}: H^{\mathrm{N}}\left(N^{\varphi} ; X\right) \rightarrow H^{\mathrm{N}}\left(N ; X^{\prime}\right)$ for any $X^{\prime}$-module $N$ with a vanishing compound action, then $\varphi_{*}: H^{\mathrm{D}}\left(M^{\varphi} ; X\right) \rightarrow H^{\mathrm{D}}\left(M ; X^{\prime}\right)$ is an isomorphism.

Proof Consider the following diagram with exact rows:

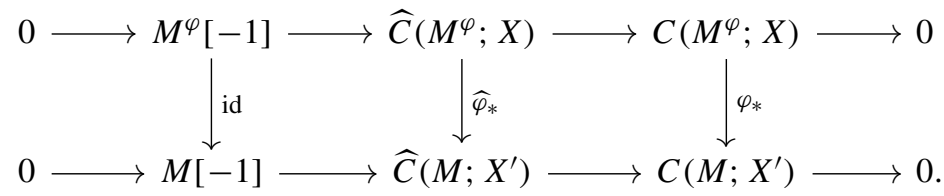

Due to the 5-lemma, $\widehat{\varphi}_{*}$ induces an isomorphism on homology if and only if so does $\varphi_{*}$.

Denote by $E^{r}$ and $\bar{E}^{r}$ the spectral sequences for $H^{\mathrm{D}}\left(M^{\varphi} ; X\right)$ and $H^{\mathrm{D}}\left(M ; X^{\prime}\right)$. Since $\widehat{H}_{k}\left(M^{\varphi} ; X\right)=\widehat{H}_{k}^{\mathrm{N}}\left(M^{\varphi} ; X\right)$ and $\widehat{H}_{k}\left(M ; X^{\prime}\right)=\widehat{H}_{k}^{\mathrm{N}}\left(M ; X^{\prime}\right)$ for $k<1$, we immediately see that $f_{p q}^{2}: E_{p q}^{2} \rightarrow \bar{E}_{p q}^{2}$ is an isomorphism for $q<3$ (homology is a module with a vanishing compound action). It follows now from Lemma 8.8 that $f_{p q}^{\infty}$ is an isomorphism if $p+q=1$, so that $H_{1}^{\mathrm{D}}\left(M^{\varphi} ; X\right) \cong H_{1}^{\mathrm{D}}\left(M ; X^{\prime}\right)$. Hence, $f_{p q}^{2}$ is an isomorphism for $q<4$ and similarly we get $H_{2}^{\mathrm{D}}\left(M^{\varphi} ; X\right) \cong H_{2}^{\mathrm{D}}\left(M ; X^{\prime}\right)$. Use induction to finish the proof.

In the next two sections we shall strengthen this result for the one-term and rack homology, obtaining recursive formulas for degenerate homology in terms of the normalized one.

\section{Degenerate spindle homology}

The filtration $\mathcal{F}^{p}$ of $C^{\mathrm{D}}(M ; X)$ is given on generators, so that the chain groups gr $C_{n}^{\mathrm{D}}(M ; X)$ and $C_{n}^{\mathrm{D}}(M ; X)$ are naturally isomorphic. We shall identify them together. However, this identification is not compatible with differentials, and our goal is to find correcting terms to obtain a chain map $f: \operatorname{gr} C_{n}^{\mathrm{D}}(M ; X) \rightarrow C_{n}^{\mathrm{D}}(M ; X)$ that is filtered, i.e. we require $f$ to send $\operatorname{gr}_{p} C^{\mathrm{D}}(M ; X)$ into $\mathcal{F}^{p}$. The following lemma is a classical result from the theory of filtered modules [9]. 
Lemma 6.1 Let $C$ and $D$ be chain complexes with filtrations $\mathcal{F}^{p} C$ and $\mathcal{F}^{p} D$ respectively, and let $f: C \rightarrow D$ be a filtered chain map, i.e. $f\left(\mathcal{F}^{p} C\right) \subset \mathcal{F}^{p} D$ for any $p$. If gr $f: \operatorname{gr} C \rightarrow \operatorname{gr} D$ is an isomorphism, so is $f$.

Corollary 6.2 A filtered chain map $f: \operatorname{gr} C^{\mathrm{D}}(M ; X) \rightarrow C^{\mathrm{D}}(M ; X)$ is an isomorphism if and only if $\mathrm{gr}_{p} C^{\mathrm{D}}(M ; X) \stackrel{f}{\rightarrow} \mathcal{F}^{p} \stackrel{\mathrm{pr}}{\rightarrow} \mathcal{F}^{p} / \mathcal{F}^{p-1}$ is an isomorphism for every $p$.

We define $\mathcal{E}: \operatorname{gr} C^{\mathrm{D}}(M ; X) \rightarrow C^{\mathrm{D}}(M ; X)$ by correcting the identity homomorphisms with lower order terms. Namely, the component $\mathcal{E}^{p}: \operatorname{gr}_{p} C^{\mathrm{D}}(M ; X) \rightarrow \mathcal{F}^{p}$ is given by the following picture:

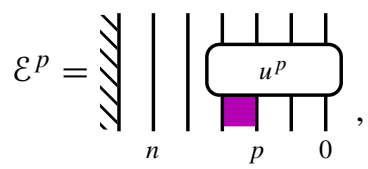

where the map $u^{p}: C_{p+1}(X) \rightarrow C_{p+1}(X)$ is defined using the recursive formula

$$
u^{0}=|| u^{p}=\stackrel{11}{u^{p-1}} \mid+(-1)^{p}
$$

Theorem 6.3 The map $\mathcal{E}: \operatorname{gr} C^{\mathrm{D}}\left(M ; X, \partial^{\star}\right) \rightarrow C^{\mathrm{D}}\left(M ; X, \partial^{\star}\right)$ is a natural isomorphism of filtered complexes. In particular, there is a natural isomorphism

$$
H_{n}^{\mathrm{D}}\left(M ; X, \partial^{\star}\right) \cong \bigoplus_{p+q=n} \widehat{H}_{q-2}\left(M ; X, \partial^{\star}\right) \otimes C_{p}^{\mathrm{N}}(X)
$$

for any spindle $(X, \star)$.

Proof Naturality of $\mathcal{E}$ is clear from the way it is constructed, and we shall show it is a chain map. First, observe that we can pull $u^{p}$ through a line:

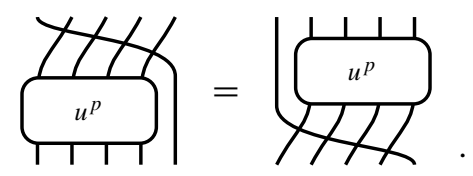

This follows directly from (7) by induction on the number of strands. In particular, we can pull the right most line to the left either over or below $u^{p-1}$ in (7). Then a simple induction shows each component $\mathcal{E}^{p}$ is a chain map:

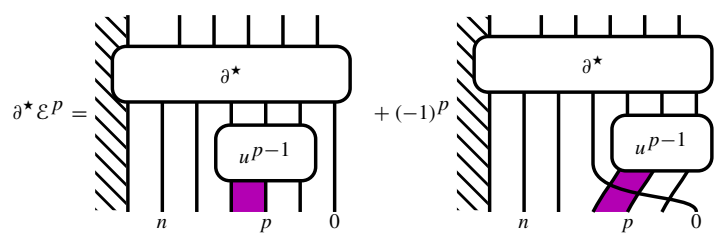




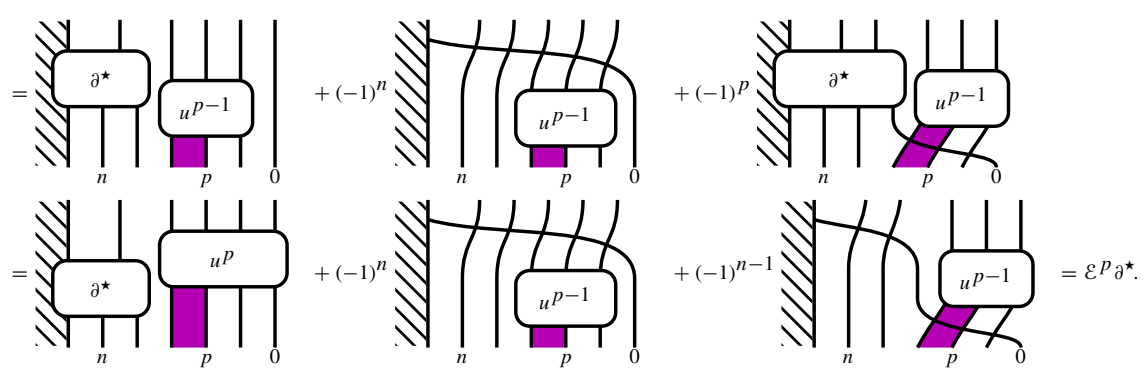

Now, the theorem follows from Corollary 6.2, as $\mathcal{E}^{p}(m \otimes \underline{x}) \in m \otimes \underline{x}+F^{p-1}$.

According to the theorem above, every degenerate homology group splits into a direct sum of copies of whole homology groups in lower degrees. These in turn split canonically into normalized and degenerate parts and the latter can be recursively replaced with groups in lower degrees.

Corollary 6.4 If the spindle $(X, \star)$ is finite,

$$
H_{n}^{\mathrm{D}}\left(X, \partial^{\star}\right) \cong \bigoplus_{p=1}^{n} \widetilde{H}_{n-p}^{\mathrm{N}}\left(X, \partial^{\star}\right)^{\oplus|X|\left(|X|^{p}-(-1)^{p}\right) /(1+|X|)} .
$$

Proof We proof the formula by induction on $n$. It is clear for $n<2$, as both sides are trivial $\left(H_{1}^{\mathrm{D}}\left(X, \partial^{\star}\right)=0\right.$, since $(x, x)=\partial^{\star}(x, x, x)$ for any $\left.x \in X\right)$. For bigger $n$ notice first that $\operatorname{rk} C_{p}^{\mathrm{N}}(X)=|X|(|X|-1)^{p-1}$. In particular, we have $\operatorname{rk} C_{p+1}^{\mathrm{N}}(X)=$ $(|X|-1) \operatorname{rk} C_{p}^{\mathrm{N}}(X)$ and Theorem 6.3 implies

$$
\begin{aligned}
H_{n+1}^{\mathrm{D}}\left(X, \partial^{\star}\right) & \cong \widetilde{H}_{n-1}\left(X, \partial^{\star}\right)^{\oplus|X|} \oplus \bigoplus_{p=2}^{n} \widetilde{H}_{n-p}\left(X, \partial^{\star}\right)^{\oplus|X|(|X|-1)^{p-1}} \\
& \cong \widetilde{H}_{n-1}^{\mathrm{N}}\left(X, \partial^{\star}\right)^{\oplus|X|} \oplus H_{n-1}^{\mathrm{D}}\left(X, \partial^{\star}\right)^{\oplus|X|} \oplus H_{n}^{\mathrm{D}}\left(X, \partial^{\star}\right)^{\oplus(|X|-1)}
\end{aligned}
$$

Using induction we show that $\widetilde{H}_{n-1}^{\mathrm{N}}\left(X, \partial^{\star}\right)$ does not appear in the second nor the third terms, and $\widetilde{H}_{n-2}^{\mathrm{N}}\left(X, \partial^{\star}\right)$ comes only from the last summand in multiplicity $|X|(|X|-1)$. For $p>2$, the group $\widetilde{H}_{n+1-p}^{\mathrm{N}}\left(X, \partial^{\star}\right)$ appears with multiplicity

$$
\begin{aligned}
\frac{|X|}{1+|X|}\left(|X|\left(|X|^{p-2}-(-1)^{p}\right)+(|X|\right. & \left.-1)\left(|X|^{p-1}+(-1)^{p}\right)\right) \\
& =\frac{|X|}{1+|X|}\left(|X|^{p}-(-1)^{p}\right)
\end{aligned}
$$

as desired.

The above corollary shows that the size of $H^{\mathrm{D}}(X)$ is determined by $H^{\mathrm{N}}(X)$ and the size of the spindle $X$. In fact, $H^{\mathrm{D}}(X)$ determines $X$ unless $\widetilde{H}^{\mathrm{N}}(X)=0$. 
Proposition 6.5 Suppose $\widetilde{H}_{p}^{\mathrm{N}}\left(X, \partial^{\star}\right) \neq 0$ for some $p$. Then a spindle homomorphism $\varphi: X \rightarrow X^{\prime}$ induces an isomorphism on degenerate homology if and only if it is an isomorphism of spindles.

Proof Choose the smallest $p$ for which $\widetilde{H}_{p}^{\mathrm{N}}\left(X, \partial^{\star}\right) \neq 0$. Then

$$
H_{p+1}^{\mathrm{D}}\left(X, \partial^{\star}\right) \cong \widetilde{H}_{p}^{\mathrm{N}}\left(X, \partial^{\star}\right) \otimes C_{0}^{\mathrm{N}}\left(X, \partial^{\star}\right)
$$

and the map induced by $\varphi$ has the form

$$
\varphi_{*}: H_{p+1}^{\mathrm{D}}\left(X, \partial^{\star}\right) \rightarrow H_{p+1}^{\mathrm{N}}\left(X^{\prime}, \partial^{\star}\right), \quad(\ldots) \otimes x \mapsto(\ldots) \otimes \varphi(x) .
$$

Hence, $\varphi$ must be bijective if $\varphi_{*}$ is an isomorphism.

\section{Degenerate generalized rack homology}

In the case of the rack differential $\partial^{\mathrm{R}}=\partial^{\vdash}-\partial^{\star}$ the degenerate complex is no longer isomorphic to $\operatorname{gr} C^{\mathrm{D}}(M ; X)$. Instead we shall show it is isomorphic to the total complex of the bicomplex $B_{p q}(M ; X)=\widehat{C}_{q-2}(M ; X) \otimes C_{p}^{\mathrm{N}}(X)$. We filter the bicomplex $B(M ; X)$ by columns, i.e. $\mathcal{F}^{p} B=\widehat{C}(M ; X) \otimes C_{p}^{\mathrm{N}}(X)$.

Due to Corollary 5.2, the chain modules $B_{p q}(M ; X)$ are isomorphic to the quotients $\mathcal{F}_{p+q}^{p} / \mathcal{F}_{p+q}^{p-1}$, which can be seen as subgroups of $C_{p+q}^{\mathrm{D}}(M ; X)$. More precisely, there is a family of monomorphisms $i_{p q}: B_{p q}(M ; X) \rightarrow C_{p+q}^{\mathrm{D}}(M ; X)$ defined as $i_{p q}(m \otimes \underline{x} \otimes \underline{y})=m \otimes \underline{x} \otimes s(\underline{y})$ and visualized by thickening the $p$-th strand:

$$
i_{p q}=\left.(-1)^{p} \searrow||\left|\bigcup_{p}\right|\right|_{0} .
$$

Recall that doubling the left most element is coherent with the rack differential, i.e. ${ }_{\partial}{ }^{\mathrm{R}}(\underline{x})=s\left(\partial^{\mathrm{R}} \underline{x}\right)$. We now define a family of maps $\mathcal{E}^{p}: \mathcal{F}^{p} B \rightarrow C_{p+q}^{\mathrm{D}}(M ; X)$ by composing $i_{p}$ with the map $u^{p}: C_{p+1}(X) \rightarrow C_{p+1}(X)$ defined in the previous section and the splitting homomorphism $\alpha: C^{\mathrm{N}}(X) \rightarrow C(X)$ acting on the normalized factor:

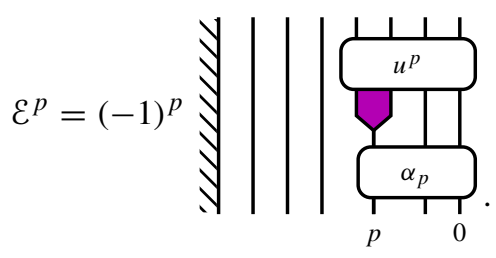

The homomorphism $\alpha_{p}$ translates the normalized rack differential into the unnormalized one, which makes graphical calculus easier-we do not have to care about repetitions when applying $u^{p}$. 
Theorem 7.1 The map $\mathcal{E}: \operatorname{Tot}(B(M ; X)) \rightarrow C^{\mathrm{D}}(M ; X)$ is an isomorphism offiltered complexes. In particular, if $R$ is a p.i.d. there is a short exact sequence

$$
\begin{array}{r}
0 \longrightarrow \bigoplus_{p+q=n} \widehat{H}_{q-2}\left(M ; X, \partial^{\mathrm{R}}\right) \otimes H_{p}^{\mathrm{N}}\left(X, \partial^{\mathrm{R}}\right) \longrightarrow H_{n}^{\mathrm{D}}\left(M ; X, \partial^{\mathrm{R}}\right) \\
\longrightarrow \bigoplus_{p+q=n-1} \operatorname{Tor}\left(\widehat{H}_{q-2}\left(M ; X, \partial^{\mathrm{R}}\right), H_{p}^{\mathrm{N}}\left(X, \partial^{\mathrm{R}}\right)\right) \longrightarrow 0
\end{array}
$$

which splits.

Proof As before, $\mathcal{E}^{p}(\underline{x}) \in \underline{x}+F^{p-1}$, which in view of Corollary 6.2 guarantees that $\mathcal{E}$ is an isomorphism if it is a chain map. This follows immediately from Theorem 6.3. Indeed,

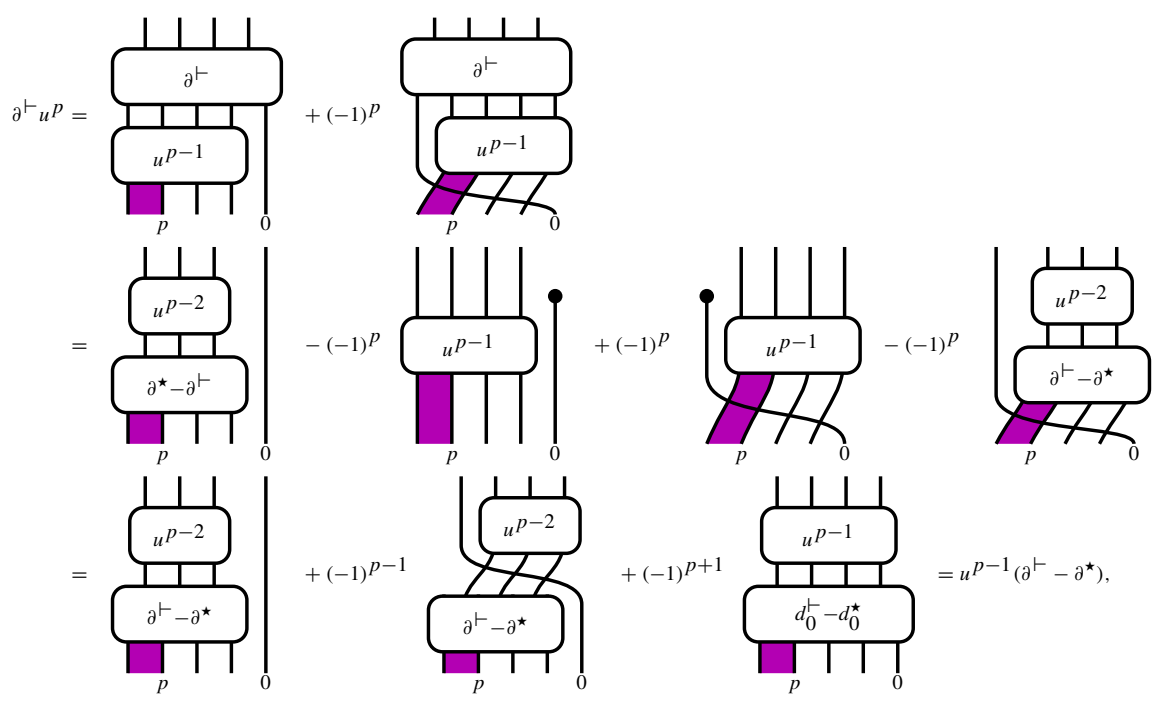

and together with $\partial^{\mathrm{R}} s \alpha=s \alpha \bar{\partial}^{\mathrm{R}}$ it implies ${ }^{2}$

$$
\partial^{\mathrm{R}} \mathcal{E}^{p}(a \otimes b)=\mathcal{E}^{p}\left(\partial^{\mathrm{R}} a \otimes b\right)+(-1)^{q} \mathcal{E}^{p}\left(a \otimes \bar{\partial}^{\mathrm{R}} b\right)
$$

for $a \in \widehat{C}_{q}(M ; X)$ and $b \in C_{p}^{\mathrm{N}}(X)$. The existence of a short exact sequence follows from the Künneth theorem.

Corollary 7.2 Suppose a spindle homomorphism $\varphi: X \rightarrow X^{\prime}$ induces an isomorphism on normalized rack homology $\varphi_{*}: H^{\mathrm{N}}\left(X, \partial^{\mathrm{R}}\right) \rightarrow H^{\mathrm{N}}\left(X^{\prime}, \partial^{\mathrm{R}}\right)$. If $M$ is an $X^{\prime}$-module such that the induced map $\varphi_{*}: H^{\mathrm{N}}\left(M^{\varphi} ; X, \partial^{\mathrm{R}}\right) \rightarrow H^{\mathrm{N}}\left(M ; X^{\prime}, \partial^{\mathrm{R}}\right)$ is also an isomorphism, so is $\varphi_{*}: H^{\mathrm{D}}\left(M^{\varphi} ; X, \partial^{\mathrm{R}}\right) \rightarrow H^{\mathrm{D}}\left(M ; X^{\prime}, \partial^{\mathrm{R}}\right)$.

\footnotetext{
${ }^{2}$ For clarity we used here $\bar{\partial}^{\mathrm{R}}$ for the rack differential in the normalized complex, to distinguish it from the unnormalized one.
} 
Proof Use a similar induction argument to the one from the proof of Theorem 5.8 to show that $B\left(M^{f} ; X\right) \cong B\left(M ; X^{\prime}\right)$.

Remark 7.3 The proof can be easily extended to the case $\partial^{a, b}=a \partial^{\star}+b \partial^{\vdash}$, when one replaces $B(M ; X)$ with the bicomplex $\widehat{C}\left(M ; X, \partial^{a, b}\right)[2] \otimes C^{\mathrm{N}}\left(X, b \cdot \partial^{\mathrm{R}}\right)$. In particular, $H^{\mathrm{D}}\left(X, \partial^{a, b}\right)$ is fully determined by the rack-type homology $H^{\mathrm{N}}\left(X, b \cdot \partial^{\mathrm{R}}\right)$, which can be computed from $H^{\mathrm{N}}\left(X, \partial^{\mathrm{R}}\right)$; see [11, Lemma 5.3] for a more detailed statement.

\section{Appendix: Sectral sequences}

This section provides basic definitions and results on spectral sequences. Most theorems are left without proofs, which can be found for example in [9]. The exception is Lemma 8.8, which seems to be unknown. It is a key ingredient to the proof of Theorem 5.8.

Definition 8.1 A (homological) spectral sequence is a sequence of bigraded $R$ modules $\left\{E^{r}\right\}_{r \in \mathbb{N}}$ together with differentials $d^{r}: E^{r} \rightarrow E^{r}$, each of degree $(-r, r-1)$, such that $H_{*}\left(E^{r}, d^{r}\right) \cong E^{r+1}$. The chain complex $\left(E^{r}, d^{r}\right)$ is called the $r$-th page of the spectral sequence $(E, d)$.

Definition 8.2 A morphism of spectral sequences $f: E \rightarrow \bar{E}$ is a sequence of module homomorphisms $f^{r}: E^{r} \rightarrow \bar{E}^{r}$ such that $f^{r+1}$ is equal to the induced homomorphism $f_{*}^{r}: H\left(E^{r}, d^{r}\right) \rightarrow H\left(\bar{E}^{r}, d^{r}\right)$.

We shall be interested only in the so-called first quadrant spectral sequences, i.e. those with $E_{p q}^{r}=0$ if $p<0$ or $q<0$. Then it must be $E_{p q}^{r}=E_{p q}^{r+1}$ for $r>p+q+1$, which means the sequence converges; we shall write $E_{p q}^{\infty}$ for the limit groups. A natural source of such spectral sequences is provided by filtered chain complexes.

Definition 8.3 A filtration of a graded $R$-module $M$ is a sequence of graded submodules $\mathcal{F}^{0} v M \subset \mathcal{F}^{1} M \subset \mathcal{F}^{2} M \subset \cdots$ such that $\bigcup_{i \in \mathbb{N}} \mathcal{F}^{i} M=M$. We say the filtration is bounded if for every $n \in \mathbb{Z}$ there exists $i \in \mathbb{N}$ such that $M_{n}=\mathcal{F}^{i} M_{n}$.

Given a filtered graded $R$-module $(M, \mathcal{F})$, we define its graded associated module as

$$
\operatorname{gr} M=\bigoplus_{i \in \mathbb{N}} \mathcal{F}^{i+1} M / \mathcal{F}^{i} M
$$

Definition 8.4 A spectral sequence $\left\{E^{r}, d^{r}\right\}_{r \in \mathbb{N}}$ converges to a filtered module $M$ if it stabilizes and $E_{p q}^{\infty} \cong \mathrm{gr}_{p} M_{q}$.

Given a filtered chain complex $(C, \mathcal{F})$, we filter its homology by images of homology of the subcomplexes: $\mathcal{F}^{p} H(C)=\operatorname{im}\left(H\left(\mathcal{F}^{p} C\right) \rightarrow H(C)\right)$. Clearly, $\mathcal{F} H$ is bounded if so is $\mathcal{F} C$.

Theorem 8.5 (cf. [9]) Let $(C, \mathcal{F})$ be a chain complex with a bounded filtration. Then there exists a spectral sequence $\left(E^{r}, d^{r}\right)$ with $E_{p q}^{1}=H_{p+q}\left(\mathcal{F}^{p} C / \mathcal{F}^{p-1} C\right)$ converging to $H(C)$. 
Given filtered chain complexes $(C, \mathcal{F})$ and $\left(C^{\prime}, \mathcal{F}\right)$, we say a chain map $f: C \rightarrow$ $C^{\prime}$ is filtered if $f\left(\mathcal{F}^{p} C\right) \subset \mathcal{F}^{p} C^{\prime}$ for any $p$. Such a map induces a morphism on the associated quotients $f_{*}: \mathcal{F}^{p} C / \mathcal{F}^{p-1} C \rightarrow \mathcal{F}^{p} C^{\prime} / \mathcal{F}^{p-1} C^{\prime}$ and a homomorphism of spectral sequences $f^{r}: E^{r} \rightarrow \bar{E}^{r}$ (the associated quotients form the 0 -th page).

Theorem 8.6 (cf. [9]) Assume $f:(C, \mathcal{F}) \longrightarrow\left(C^{\prime}, \mathcal{F}\right)$ is a filtered chain map and the filtrations on $C$ and $C^{\prime}$ are bounded. Denote by $E^{r}$ and $\bar{E}^{r}$ the spectral sequences for $(C, \mathcal{F})$ and $\left(C^{\prime}, \mathcal{F}\right)$ respectively. If $f_{p q}^{\infty}: E_{p q}^{\infty} \rightarrow \bar{E}_{p q}^{\infty}$ is an isomorphism for all $p+q=n$, then so is $f_{*}: H_{n}(C) \rightarrow H_{n}\left(C^{\prime}\right)$.

In other words, if $f$ induces an isomorphism on graded modules associated to homology, then the map of homology is also an isomorphism. In fact, it is enough to have an isomorphism at certain page to deduce that homology of $C$ and homology of $C^{\prime}$ are isomorphic.

Corollary 8.7 In the situation as above assume that $f^{r}: E^{r} \rightarrow \bar{E}^{r}$ is an isomorphism for some $r \in \mathbb{N}$. Then $f_{*}: H(C) \rightarrow H\left(C^{\prime}\right)$ is an isomorphism.

However, we need a weaker result with $f^{r}$ being an isomorphism only in certain degrees.

Lemma 8.8 Assume there is a homomorphism of spectral sequences $f_{p q}^{r}: E_{p q}^{r} \rightarrow$ $\bar{E}_{p q}^{r}$ such that $f_{p q}^{2}$ is an isomorphism for $q \leqslant N$. Then $f_{p q}^{\infty}: E_{p q}^{\infty} \rightarrow \bar{E}_{p q}^{\infty}$ is an isomorphism for $p+q=N$.

The main idea is that if a chain map $g_{i}: C_{i} \rightarrow C_{i}^{\prime}$ between two chain complexes is an isomorphism for $i=i_{0}, i_{0}-1$, and $i_{0}+1$, then $g_{*}: H_{i}(C) \rightarrow H_{i}\left(C^{\prime}\right)$ is an isomorphism. Indeed, let $t_{i} C$ be the subquotient of $C$ with $\left(t_{i} C\right)_{j}=C_{j}$ whenever $j \in\{i-1, i, i+1\}$ and zero otherwise. Likewise we define $t_{i} C^{\prime}$. Then $H_{i}\left(t_{i} C\right) \cong$ $H_{i}(C)$ and similarly for $C^{\prime}$, whereas $g: C \rightarrow C^{\prime}$ descends to an isomorphism between $t_{i} C$ and $t_{i} C^{\prime}$.

Therefore, as $f_{p q}^{2}$ is an isomorphism for $q \leqslant N$, and the differential $d^{2}$ increases $q$ by 1 and decreases $p$ by $2, f_{p q}^{3}$ is an isomorphism whenever $q \leqslant N-1$ or $q=N$ and $p<2$. The next differential, $d^{3}$, increases $q$ by 2 and decreases $p$ by 3 . Hence, $f_{p q}^{4}$ is an isomorphism for $q \leqslant N-3$ and a few more points: $p<2$ and $q \leqslant N$, or $p=2$ and $q \leqslant N-1$, or $p=3,4$ and $\leqslant N-2$. By repeating this process we see, that the set of points $(p, q)$ for which $f_{p q}^{r}$ is an isomorphism from a staircase diagram, see Fig. 2. We want to show that the whole diagonal $p+q=N$ is below the stairs for any $r$.

Proof of Lemma 8.8 Define functions $p^{i}(r)=\sum_{k=1}^{i}(r-k)$. In particular, we have

$$
p^{i}(r+1)=p^{i}(r)+i, \quad p^{i+1}(r+1)=p^{i}(r)+r .
$$

Let $\mathcal{J}^{r} \subset \mathbb{N} \times \mathbb{N}$ consist of pairs $(p, q)$ satisfying the following condition:

$$
\begin{cases}q \leqslant N, & \text { if } p=0, \\ q \leqslant N+i-p, & \text { if } p^{i-1}(r)<p \leqslant p^{i}(r) \text { for some } i \\ q \leqslant N+(r-2)-p^{r-2}(r), & \text { if } p>p^{r-2}(r) .\end{cases}
$$




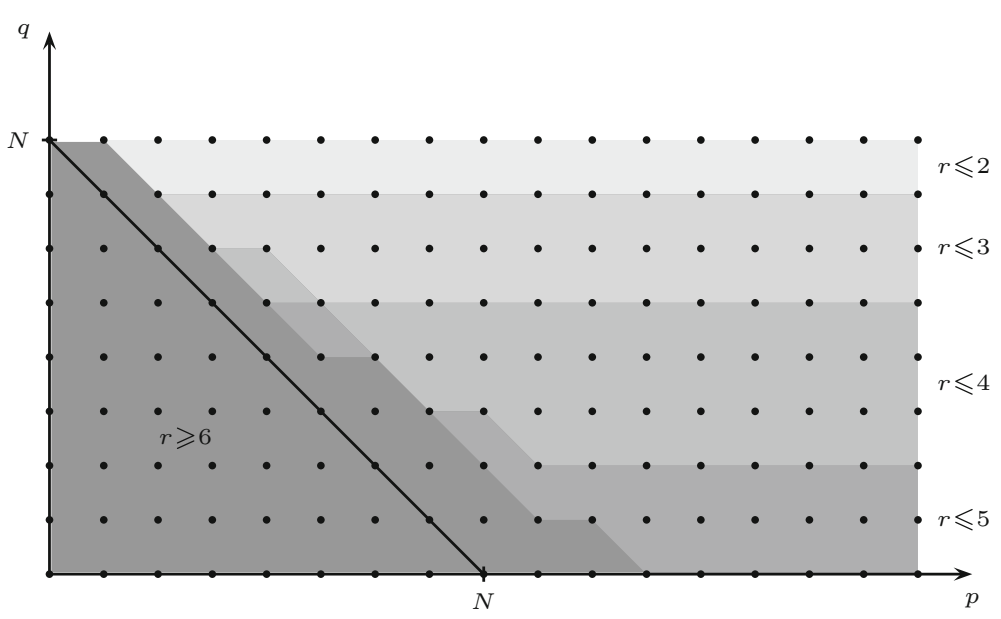

Fig. 2 The sets $\mathcal{J}^{r}$ of points $(p, q)$ at which $f_{p q}^{r}$ is an isomorphism, visualized for $N=8$. A region labeled $r \leqslant i$ belongs to every $\mathrm{J}^{r}$ with $r \leqslant i$. The skew line represents the diagonal $p+q=N$

Notice that all $\mathrm{J}^{r}$ contain the diagonal $p+q=N$, and $f_{p q}^{2}$ is an isomorphism for $(p, q) \in \mathcal{J}^{2}$. We shall show by induction that $f_{p q}^{r+1}$ is also an isomorphism whenever $(p, q) \in \mathcal{J}^{r+1}$. For that we have to check that $f_{p q}^{r}, f_{p+r, q-r+1}^{r}$, and $f_{p-r, q+r-1}^{r}$ are isomorphisms, as $d^{r}$ lowers $p$ by $r$ and increases $q$ by $r-1$.

- Since $p^{i}(r)<p^{i}(r+1)$, we have $\mathcal{J}^{r+1} \subset \mathcal{J}^{r}$ and $(p, q) \in \mathcal{J}^{r}$. Hence, $f_{p q}^{r}$ is an isomorphism.

- Clearly, $f_{p+r, q-r+1}^{r}$ is an isomorphism if $q<r-1$ or $p>p^{r-1}(r+1)$ (the last case in (8)). Assume then that $p^{i}(r+1) \leqslant p<p^{i+1}(r+1)$ for some $i$. Then $p+r>p^{i+1}(r+1)=p^{i}(r)+r>p^{i+1}(r)$, and $(p+r)+(q-r+1)=$ $p+q+1 \leqslant N+i+1$, so that $(p+r, q-r+1) \in \mathcal{J}^{r}$.

- Finally, $f_{p-r, q+r-1}^{r}$ is an isomorphism if $p<r$, so that we can assume $p \geqslant$ $p^{i}(r+1)$ for some $i>0\left(\right.$ as $\left.p^{1}(r+1)=r\right)$. There are two subcases to check.

Case $1 p>p^{r-1}(r+1)$. We have

$$
q+r-1 \leqslant N+2(r-1)-p^{r-1}(r+1)=N+(r-2)-p^{r-2}(r),
$$

which guarantees $(p-r, q+r-1) \in \mathcal{J}^{r}$.

Case $2 p^{i}(r+1)<p \leqslant p^{i+1}(r+1)$ for some $i=0, \ldots, r-1$ (case $i=0$ happens if $p=r)$. Then $p^{i-1}(r)<p-r \leqslant p^{i}(r)$ and since

$$
(p-r)+(q+r-1)=p+q-1 \leqslant N+i,
$$

we have $(p-r, q+r-s 1) \in \mathcal{J}^{r}$.

Hence, $f_{p q}^{r+1}$ is an isomorphism if $(p, g) \in \mathcal{J}^{r}$, and so is $f_{p q}^{\infty}$ for $p+q \leqslant N$. 


\section{References}

1. Carter, J.S.: A survey of quandle ideas. In: Kauffmann, L.H., et al. (eds.) Introductory Lectures on Knot Theory. Series on Knots and Everything, vol. 46, pp. 22-53. World Scientific, Hackensack (2011)

2. Carter, J.S., Elhamdadi, M., Saito, M.: Twisted quandle homology theory and cocycle knot invariants. Algebr. Geom. Topol. 2(1), 95-135 (2002)

3. Carter, J.S., Jelsovsky, D., Kamada, S., Langford, L., Saito, M.: State-sum invariants of knotted curves and surfaces from quandle cohomology. Electron. Res. Announc. Amer. Math. Soc. 5, 146-156 (1999)

4. Carter, J.S., Jelsovsky, D., Kamada, S., Saito, M.: Quandle homology groups, their Betti numbers, and virtual knots. J. Pure Appl. Algebra 157(2-3), 135-155 (2001)

5. Crans, A.S.: Lie 2-Algebras. PhD Thesis, University of California, Riverside (2004). arXiv:math.QA/0409602

6. Dehornoy, P.: Braids and Self-Distributivity. Progress in Mathematics, vol. 192. Birkhäuser, Basel (2000)

7. Fenn, R., Rourke, C., Sanderson, B.: James bundles. Proc. London Math. Soc. 89(1), 217-240 (2004)

8. Litherland, R.A., Nelson, S.: The Betti numbers of some finite racks. J. Pure Appl. Algebra 178(2), 187-202 (2003)

9. McCleary, J.: A User's Guide to Spectral Sequences. Cambridge Studies in Advanced Mathematics, vol. 58, 2nd edn. Cambridge University Press, Cambridge (2001)

10. Przytycki, J.H.: Distributivity versus associativity in the homology theory of algebraic structures. Demonstratio Math. 44(4), 823-869 (2011)

11. Przytycki, J.H., Putyra, K.K.: Homology of distributive lattices. J. Homotopy Relat. Struct. 8(1), 35-65 (2013)

12. Przytycki, J.H., Sikora, A.S.: Distributive products and their homology. Comm. Algebra 42(3), 12581269 (2014) 